\section{Ultrastructure of human mature oocytes after vitrification}

\author{
M.A. Khalili, ${ }^{1,2}$ M. Maione, \\ M.G. Palmerini, ${ }^{3}$ S. Bianchi, ${ }^{3}$ \\ G. Macchiarelli, ${ }^{3}$ S.A. Nottola'
}

'Department of Anatomy, Histology, Forensic Medicine and Orthopaedics, La Sapienza University, Rome, Italy; ${ }^{2}$ Yazd Institute for Reproductive Sciences, Shahid Sadoughi University of Medical Sciences, Yazd, Iran; ${ }^{3}$ Department of Health Sciences, University of L'Aquila, Italy

\section{Abstract}

Since the introduction of human assisted reproduction, oocyte cryopreservation has been regarded as an attractive option to capitalize the reproductive potential of surplus oocytes and preserve female fertility. However, for two decades the endeavor to store oocytes has been limited by the not yet optimized methodologies, with the consequence of poor clinical outcome or of uncertain reproducibility. Vitrification has been developed as the promising technology of cryopreservation even if slow freezing remains a suitable choice. Nevertheless, the insufficiency of clinical and correlated multidisciplinary data is still stirring controversy on the impact of this technique on oocyte integrity. Morphological studies may actually provide a great insight in this debate. Phase contrast microscopy and other light microscopy techniques, including cytochemistry, provided substantial morphofunctional data on cryopreserved oocyte, but are unable to unraveling fine structural changes. The ultrastructural damage is one of the most adverse events associated with cryopreservation, as an effect of cryo-protectant toxicity, ice crystal formation and osmotic stress. Surprisingly, transmission electron microscopy has attracted only limited attention in the field of cryopreservation. In this review, the subcellular structure of human mature oocytes following vitrification is discussed at the light of most relevant ultrastructural studies.

\section{Introduction}

Cryopreservation of the human oocytes is currently considered an attractive and useful technology to preserve fertility, although still not fully optimized. Cryostorage could be one of the few options for patients at risk of prema- ture ovarian failure due to potentially gonadotoxic chemo/radio-therapies for malignancies, bone marrow transplantation protocols or a number of severe non-cancerous systemic diseases. ${ }^{1-4}$ 0ocyte cryopreservation might be used in women of reproductive age who choose to postpone childbearing. Cryopreservation of human oocytes also represents a valid alternative to legal, moral and religious problems concerning embryo freezing in some countries. Also, it does not require the presence of a male partner, thus having great impact on assisted reproduction technology. ${ }^{5}$ However, in contrast to the preimplantation embryo cryostorage, it is quite difficult to adequately cryopreserve metaphase II (MII) human oocytes, mainly due to their large size, water content, high degree of cytoplasmic specialization and precise chromosomal arrangement. ${ }^{6}$ Freezing of immature, germinal vesicle (GV) oocytes may elude some of the problems occurring during MII oocyte freezing, particularly those related to the cryodamage of spindle and chromosomes. Nevertheless, the current inadequacy of in vitro maturation protocols represents an impediment for obtaining viable mature gametes after cryostorage of immature oocytes, thus significantly reducing the final performance of the whole procedure. ${ }^{4,7}$

Improvements in cryotechnology and modifications of conventional protocols of slow freezing have recently determined an increase of post-thaw oocyte survival, fertilization and embryo cleaving rates. ${ }^{8}$ In spite of this, the pregnancy rates and clinical outcomes are still unsatisfactory or at least contradictory. ${ }^{6}$ The cryopreservation technique of vitrification (an ultra-rapid freezing technique) offers new interesting perspectives in the field of oocyte cryopreservation, appearing less traumatic than slow freezing. ${ }^{9-11}$ Vitrification technology is also described as an easy, quick, effective, low-cost and low-risk procedure. ${ }^{9,12}$ However, due to the limited experience reported to date, further studies are needed to determine the actual efficiency and safety of this approach. ${ }^{13}$ In particular, at the cellular level, little is known about the potentially detrimental effects of this procedure and, in particular, the high concentrations of the commonly used cryoprotectants. ${ }^{4}$ In addition, some data are presented on the comparative efficiency of commercially available vitrification supports (named open and closed devices), but most concern non-human mammals. ${ }^{41,15}$ A growing attention has been recently given to reduce cross-contaminations in the non-sterile environment of liquid nitrogen freezers, particularly in open devices, in which samples to be cryostored enter in direct contact with liquid nitrogen. On the other hand, even the closed carriers cannot theoretically avoid the transmission of microorganisms in the culture
Correspondence: Dr. Stefania Annarita Nottola, Department of Anatomy, Histology, Forensic Medicine and Orthopaedics, Section of Anatomy, La Sapienza University, via Alfonso Borelli 50, 00161 Rome, Italy.

Tel. +39.06 .49918072 - Fax: +39.06 .49918082 .

E-mail: stefania.nottola@uniromal.it

Key words: oocyte, MII, vitrification, ultrastructure, TEM, human.

Acknowledgments: this paper was presented by Prof. Stefania A. Nottola at the XXXIV National Congress of the Italian Society of Histochemistry, San Benedetto del Tronto, Italy, June 7-9, 2011.

Contributions: SAN, GM, study designed and direction; MAK, literature review, manuscript writing; GM, SAN, MGP, SB, MM, contribution to morphological studies. All authors evaluated micrographs, critically revised the manuscript and approved the final version of this article.

Conflict of interests: all the authors declare there is no conflict of interest, personal, financial or otherwise, in relation of this work.

Received for publication: 17 June 2012. Accepted for publication: 2 July 2012.

This work is licensed under a Creative Commons Attribution NonCommercial 3.0 License (CC BYNC 3.0).

(C) Copyright M.A. Khalili et al., 2012

Licensee PAGEPress, Italy

European Journal of Histochemistry 2012; 56:e38 doi:10.4081/ejh.2012.e38

medium during the warming procedure due to a possible previous direct contact between the external surface of the carrier and accidentally contaminated liquid nitrogen. ${ }^{16}$ Indeed, in the clinical practice both systems (open and closed) are allowed and routinely used, if aseptic procedures are carefully followed.

Oocyte morphology, as evaluated by phasecontrast microscopy, is an important and irreplaceable predictive indicator of oocyte quality, still in use to evaluate the success of a given assisted reproduction protocol. However, simple low-resolution morphological assessment is not fully adequate to measure oocyte fertility potential and developmental competence. ${ }^{17}$ In fact, even human oocytes that survive cryopreservation and exhibit no apparent cellular damage by conventional microscopy examination may display reduced fertilizability and arrested development during the early cleavage stage. ${ }^{18}$ The polarized light microscopy (PLM) technology allows to image meiotic spindles in vivo before and after cryopreservation and it is not harmful for the oocyte. A combined approach of PLM with immunocytochemistry has been successfully applied to detect alterations in the spindle configuration. 
Several studies analyzed spindle morphology, depolymerization and recovery in cryopreserved mammal and human oocytes but the correlation between the two methods is controversial. ${ }^{19-22}$ Even if polscope can be considered an indicator of oocyte viability and could be used to help the selection of oocytes for insemination in intracytoplasmic sperm injection (ICSI) patients, immunocytochemistry associated with epifluorescence or confocal laser scanning microscopy (CLSM) provides great detail about cryoinjuries in individual fixed oocytes. Organelles highly sensitive to cryoinjuries such as meiotic spindles, ${ }^{23,24}$ cortical granules (CG), ${ }^{25}$ cytoskeleton components ${ }^{26}$ can be studied using these combined approach. Moreover, the ability of CLSM to visualize in one focal plane the fluorescence associated with multiple markers renders this instrument extremely valuable for the study of co-localization of various markers, also allowing a 3-D reconstruction. Fluorescent in situ hybridization (FISH) is routinely used to detect aneuploidy using specific fluoroprobes for chromosome 13, 16, 18, 21 and X. ${ }^{27}$ Recent advances in techniques of live cell imaging are becoming valuable tools to assess alterations in ooplasmic compartments. Intracellular calcium concentrations has been determined on fresh and thawed-warmed human oocytes, evidencing a better preservation of calcium dynamics in oocytes subjected to a close vitrification system than slow-frozen. ${ }^{28}$ The developmental competences of vitrified oocytes can also be investigated analyzing the mitochondrial pattern or variations in the mitochondrial membrane potential. Variation in clustering and distribution of mitochondria has been found in mouse-vitrified oocytes, by staining the oocytes with specific mitochondrion selective probes as Mitotracker. ${ }^{29}$

However, to have the complete morphological view of cryopreserved oocytes, transmission electron microscopy (TEM) evaluation, alone or integrated with-immunocytochemical approach, is especially effective in estimating how cooling rates and cryoprotectants affect the oocyte structural integrity. ${ }^{18,30}$ The impact of vitrification on the MII oocyte ultrastructural morphology has been investigated in several mammals such as bovines, porcine and rodents. ${ }^{26,31-33}$ However, comprehensive studies on human vitrified-warmed oocytes are scarce and conclusive evidence concerning the identification of top-quality cryopreserved human oocytes in terms of ultrastructure has been generated just recently. ${ }^{28,34-39}$ The present review was therefore aimed to identify the main subcellular targets of cryopreservation damage to assess the effectiveness of vitrification protocols on preserving the integrity of human mature oocytes.
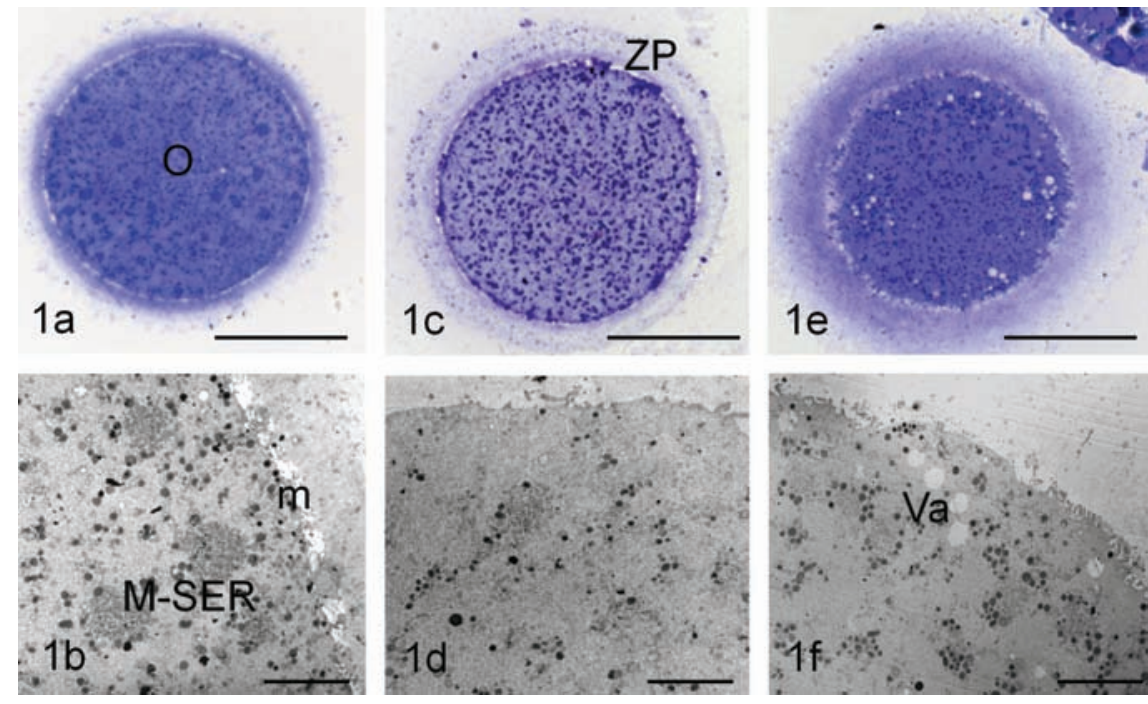

Figure 1. Fresh control $(a, b)$, vitrified-warmed $(c, d)$ and frozen-thawed by slow freezing $(e, f)$ human oocytes. The general morphology and organelle microtopography are shown by LM (a, c, e) and TEM (b, d, f). No explicit differences in shape, dimensions and organelle distribution were found between fresh and cryopreserved oocytes. Apparent variations of ZP thickness (e) is an effect of the section plane (not equatorial). Vacuoles, detected only sporadically in both fresh (a, b) and vitrified-warmed (c, d) oocytes to the same extent, were numerous in the oocytes frozen-thawed by slow cooling (e, f). m, oocyte microvilli; O, oocyte; Va, vacuoles; ZP, zona pellucida; M-SER, mitochondria-smooth endoplasmic reticulum aggregates. Scale bars: (a) $45 \mu \mathrm{m}$; (c, e) $40 \mu \mathrm{m}$; (b) $4 \mu \mathrm{m}$; (d, f) 5 um. Panels a,b,e,f: modified from Coticchio et al. ${ }^{39}$; panels c,d: modified from Nottola et al. ${ }^{34}$

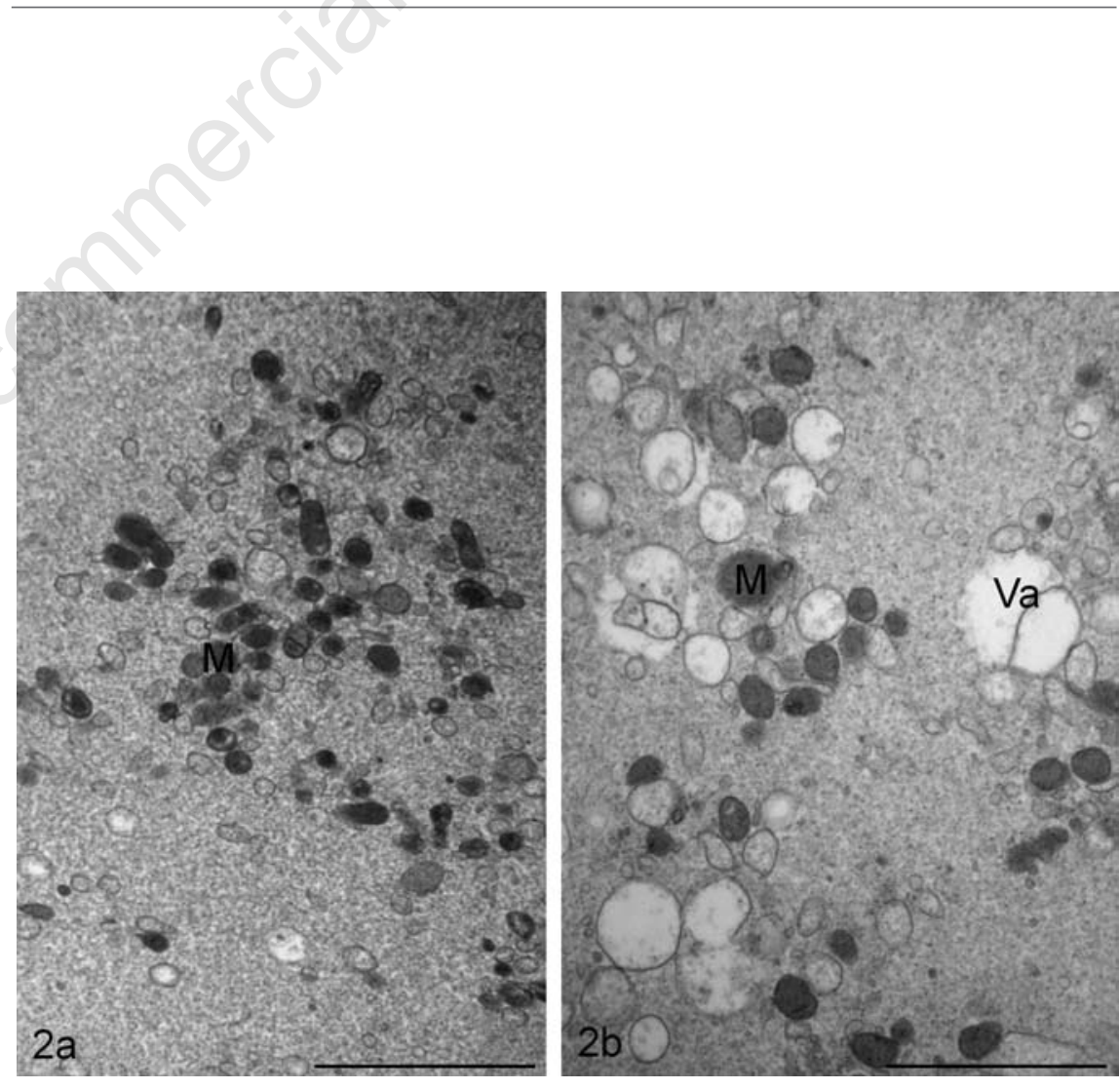

Figure 2. Vitrified-warmed (a) and frozen-thawed by slow freezing (b) human oocytes. TEM micrograph showing the paucity of vacuoles in (a) and the presence of an extensive vacuolization in (b). Va, vacuole; $M$, mitochondria. Scale bars: (a) $3 \mu \mathrm{m}$; (b) $2 \mu \mathrm{m}$. Panel b: modified from Coticchio et al. ${ }^{39}$ 


\section{Ultrastructural studies}

Electron microscopy techniques are the best tools to explore the cell ultrastructure, but involve important limitations. They rely on expensive technology and highly trained personnel. They can be hardly used for the analysis of large numbers of samples, being the process of specimen preparation and observation lengthy and almost entirely manual. Regardless, they can provide accurate and extensive information on the fine structure of the cell and its organelles. . $^{18,34}$ Sathananthan et al. were the first to report damage to the zona pellucida (ZP), oolemma, and ooplasm in cryopreserved human oocytes. ${ }^{40}$ With the exception of an analysis of Van Blerkom and Davies, in the mid-nineties, ${ }^{41}$ further evidence on the ultrastructure of frozen-thawed oocytes has been gained only recently. Ultrastructural alterations involving the CG, ZP, organelle associations, and ooplasm were reported in coincidence with the application of particular dehydration and rehydration conditions, ${ }^{25,38-41}$ suggesting that TEM can represent a first line investigative tool. Disagreement exists on the entity and type of cell damage that human oocyte may suffer as a result of cryopreservation. Several studies have reported pro and versus the hypothesis that the MII spindle can be irreversibly damaged by low temperature storage. ${ }^{42}$ During the process of freezing-thawing the oocyte is exposed to a variety of physical and chemical conditions, such as suboptimal temperatures, formation of intracellular ice crystals, osmotic stress and replacement of intracellular water with cryoprotective agents, that may endanger its survival and competence and may cause subcellular changes undetectable by low resolution microscopy. ${ }^{43}$ It appears essential to define objective criteria to establish whether oocyte quality may be affected by cryopreservation, in order to ruling out the applicability of different protocols and appraise possible health risks for children born from cryopreserved oocytes.

In our experience, to this aim, the following structural and ultrastructural characteristics of the oocyte should be especially evaluated (methods are analytically reported by Nottola et al. $^{34}$ ).

General features, e.g., shape, dimensions, density of ooplasm, integrity of GV (in immature oocyte) and, in favorable sections, the arrangement of the MII spindle (spindle reassembly and chromosome alignment).

Vacuoles, lysosomes, multivesicular bodies (MVB)

Microtopography of the organelles:

- mitochondria

mitochondria-smooth endoplasmic

reticulum (M-SER) aggregates

mitochondria-vesicle (M-V) complexes

CG pattern and ZP texture

Integrity of the oolemma

\section{General features of vitrified oocytes}

Cryopreservation does not significantly impair mammalian oocyte general microarchitecture. ${ }^{34}$ The vitrified-warmed oocytes are regular in shape. Dimensions and density of the ooplasm in light microscopy (LM) examination of semithin sections may be compared to the fresh counterpart ${ }^{34,35}$ (Figure 1). Indeed, human mature oocytes subjected to the vitrification procedures may show general ultrastructural features compatible with criteria of maturity and viability similar to those found in slow-frozen human oocytes
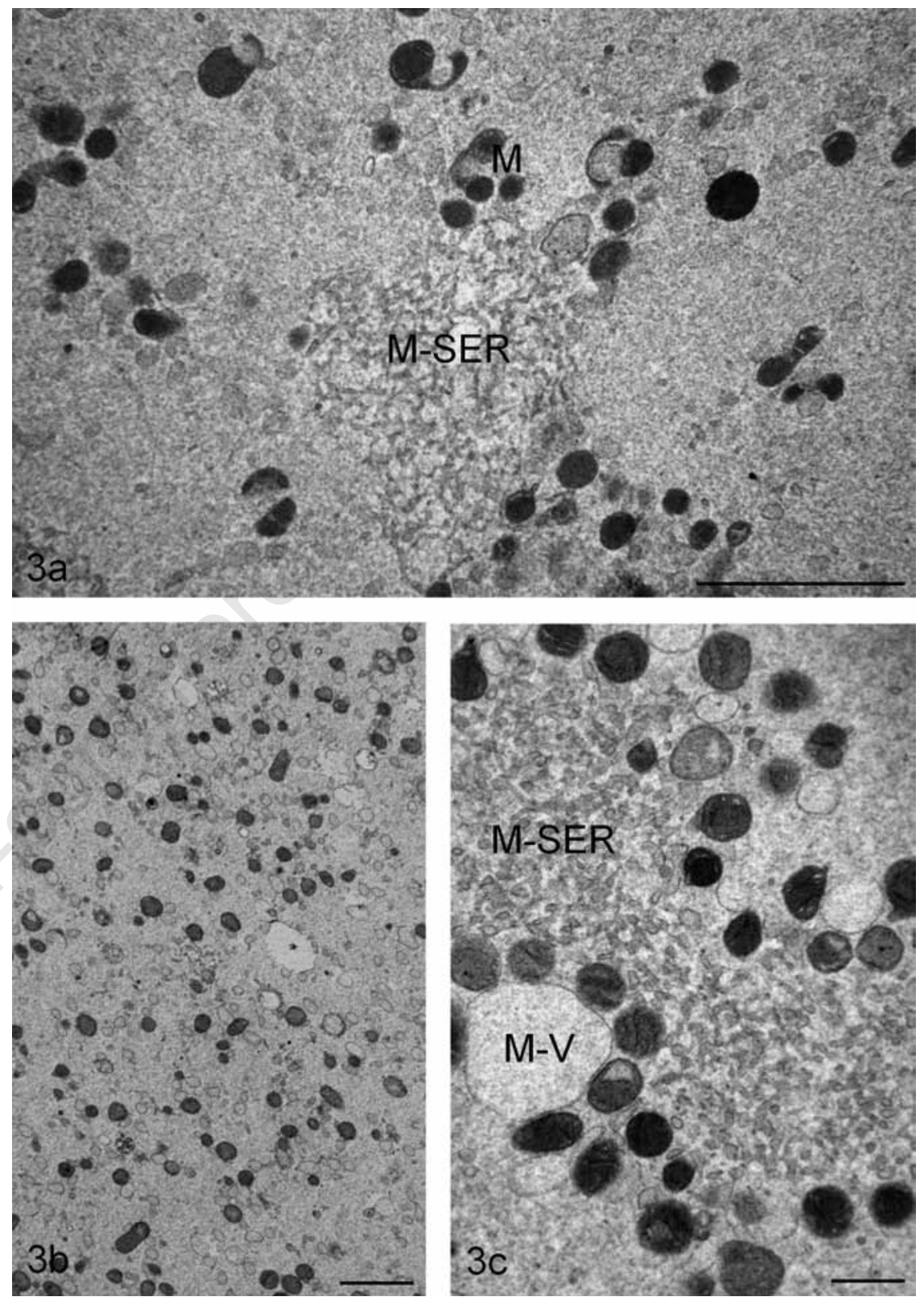

Figure 3. Fresh control (a) and vitrified-warmed (b, c) human oocytes. Mitochondria are generally rounded and provided with a few peripheral or transverse cristae. Dumb-bell shaped mitochondria (likely dividing) can be occasionally found in the ooplasm (a). Voluminous aggregates between mitochondria and smooth endoplasmic reticulum are seen $(a, c)$. Note in (b) the absence of regularly shaped M-SER aggregates in the ooplasm. M, mitochondria; M-SER, mitochondria-smooth endoplasmic reticulum aggregates; $M$ V, mitochondria-vesicle complexes. Scale bars: (a) $2.5 \mu \mathrm{m}$; (b) $2 \mu \mathrm{m}$; (c) $0.5 \mu \mathrm{m}$. Modified from Nottola et al. ${ }^{34}$ 
and in fresh oocytes as well. ${ }^{36,37,44,45}$

In immature GV-stage oocytes, there is no evidence on the presence of ultrastructural damages to the nuclear envelope, nucleoplasm, nucleolar bodies, euchromatin and heterochromatin (unpublished results). In matured oocyte, occasional detection of meiotic spindles in both fresh and cryopreserved oocytes is obtained by LM and TEM only in particularly favorable sections. In these sections, the spindle of cryopreserved oocyte appears usually well structured. As above stated, since it is not possible to obtain repetitive and systematic information on spindle morphology by means of electron microscopy alone, other morphological approaches mainly based on immunocytochemistry are needed.

\section{Vacuoles, lysosomes, multi- vesicular bodies}

A slight vacuolization of the cytoplasm is rather normal in GV oocyte and pronuclear eggs, but is very rare in MII oocytes..$^{34,46}$ Different degrees of vacuolization have been detected in MII oocytes after cryopreservation. Following vitrification it is becoming evident a cryodevice dependency to vacuolization. In fact, only rare vacuoles were occasionally detected in human MII vitrified by the open carrier devices Cryoloop and Cryoleaf ${ }^{34}$ (Figures 1c,d and 2a). On the contrary, a slight degree of vacuolization was found in human MII oocytes vitrified with CryoTip (closed device). ${ }^{28,35}$ Abundant vacuolization was instead constantly observed in human mature oocytes after slow-freezing, irrespective of the procedure applied ${ }^{25,36-39,46}$ (Figures 1e,f and 2b). Vacuolization may be a non-specific response of the oocyte to cryoinjury and/or osmotic stress. Multiple vacuoles in the ooplasm of human oocytes may be caused by swelling and coalescence of isolated SER. They are considered the morphological expression of a degenerative process, ${ }^{30,38}$ as shown by the recurrent finding of associations of vacuoles with MVB and lysosomes. ${ }^{34,36}$ MVB are considered a type of digestive organelle related to exchange processes occurring at the oocyte surface, whilst secondary lysosomes are involved in degradation of oocytic materials. However, both organelles, lysosomes in particular, may be considered as typical regressive markers when associated with vacuoles in the oocyte. ${ }^{47,4}$. Thus, as the appearance of vacuoles in human mature oocytes might ultimately lead to a reduced oocyte competence to fertilization and/or impaired embryo development, ${ }^{46}$ the quasi absence of vacuolization in the vitrified-warmed oocytes could be interpreted as a marker of a good quality of the oocyte. ${ }^{34}$
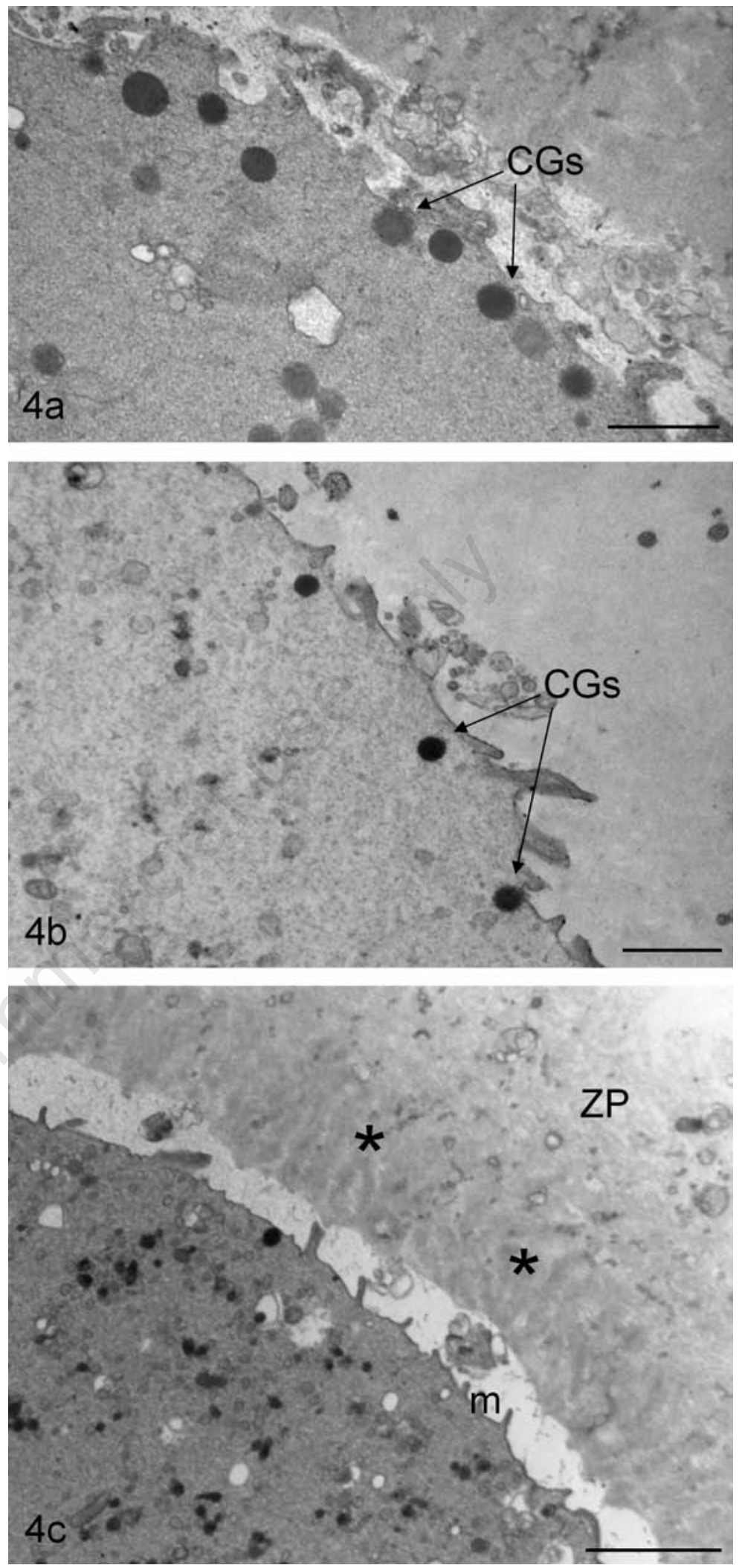

Figure 4. Fresh control (a) and vitrified-warmed (b, c) human oocytes. A rim of electrondense CG (arrows) is seen just beneath the oolemma of the fresh oocyte (a). Instead, CG are sparse and form a discontinuous layer in vitrified-warmed oocytes (b, c). Note in (c) the increased compaction of the inner aspect of the ZP (asterisks) and the reduced number of microvilli. The dense ZP in c corresponds to an area of cortical cytoplasm virtually devoid of CG. CGs, cortical granules; $m$, microvilli; ZP, zona pellucida. Scale bars: (a) $1.1 \mu \mathrm{m}$; (b) $1.3 \mu \mathrm{m}$; (c) $2.5 \mu \mathrm{m}$. Panels a,b: modified from Nottola et al. ${ }^{34}$ 


\section{Microtopography of organelles}

Human oocytes after vitrification frequently (50\%) show atypical, immature M-SER aggregates. $^{34}$ The arrangement of M-V complexes and the fine structure of mitochondria are similar in both fresh and vitrified-warmed samples $^{34}$ (Figure 3). Mitochondria show good morphological preservation after vitrification, both in mouse and human oocytes. ${ }^{33,34}$ Oocyte mitochondrial damage (e.g., modifications in shape and surface, swelling, reduced density and alteration of the cristae) has been frequently detected in some other mammals subjected to different vitrification protocols. . $^{26,31,32}$

M-SER disorganization with maintenance of mitochondrial integrity has been also found in human MII oocytes subjected to a slow freezing protocol using ethylene glycol (EG) as cryprotectant. ${ }^{37}$ Thus, the alterations in shape and/or size of M-SER aggregates observed in the vitrifiedwarmed oocytes, even in the absence of clear mitochondrial alterations, were related more to the use of EG in the vitrification solutions than to the cryodamage itself. EG is widely used in vitrification procedures because of its lower toxicity if compared with other cryoprotectants. ${ }^{49}$ However, when two solutions containing different concentrations of EG were tested, a higher proportion of human oocytes showing normal morphological features was associated with the use of the vitrification solution containing a lower EG concentratio. ${ }^{9}$

M-SER aggregates are precursor of M-V complexes. ${ }^{50}$ Mitochondria and associated cytoplasmic membranes may play a role in the neoformation of membranes during early embryogenesis. ${ }^{51} \mathrm{M}-\mathrm{SER}$ aggregates may also regulate local concentrations of free calcium and ATP production, thus acting on different cellular activities including mediation of calcium-dependent signal transduction pathways at fertilization. ${ }^{52,53}$ The ultrastructural changes in the arrangement of M-SER aggregates could be, among other factors, potential determinants of a reduced oocyte competence for fertilization, possibly due to disturbances in calcium homeostasis. It should be also considered that variations in shape and size of M-SER aggregates, in the absence of detectable damage in both SER tubules and mitochondria, could be related to different morphodynamic stages of these structures.

\section{Cortical granules pattern and texture of zona pellucida}

The number of CG may be abnormally reduced in vitrified-warmed oocytes ${ }^{34}$ (Figure 4). A compaction of the inner aspect of the ZP, with the loss of its typical filamentous texture ${ }^{54}$
(Figure 4c), due to the presence of large areas of filaments packed together, was sometimes found in the same samples. These are ultrastructural markers of the occurrence of a premature exocytosis of the CG content into the perivitelline space (PVS), with the resulting hardening of the inner aspect of ZP. Such hardening may halt sperm penetration at fertilization and this problem can be overcome only by ICSI. ${ }^{4}$ CG pattern changes were found not only in vitrified oocytes, in human and other mammals, but also in oocytes subjected to different protocols of slow freezing. ${ }^{25,31-34,36-39}$ Thus, CG exocytosis, a phenomenon that indicates a possibility of illegitimate oocyte activation, does not seem to be dependent upon the procedure applied or to the cryoprotectant used, but it is a quite common phenomenon occurring during oocyte cryopreservation, although some studies reported contrasting results. ${ }^{3,55}$

\section{Integrity of the oolemma}

Vitrified-warmed oocytes are surrounded by an intact, continuous oolemma and mostly provided with microvilli projecting into a PVS with regular shape, width and content ${ }^{30,48,50}$ ) (Figure 4c). A fraction (about 30\%) of the warmed oocytes, however, showed focal surface areas with rare and/or short microvilli. ${ }^{34}$ Similar ultrastructural features have been found in human oocytes subjected to a slow freezing protocol based on the use of EG. ${ }^{37}$ Conversely, a normal pattern of microvilli was detected in human slow-frozen oocytes cryopreserved with propane1,2-diol. ${ }^{36}$ Structural damage of microvilli in cryopreserved oocytes could be related to a primary alteration of the sub-oolemmal cytoskeletal web occurring during cryopreservation. ${ }^{33}$ The improper microvilli distribution may ultimately contribute to fertilization failure, due to ineffective spermatozoon-oocyte fusion. ${ }^{56}$

\section{Conclusions}

Human mature oocytes subjected to the vitrification show good overall ultrastructural preservation. In particular, the quasi absence of cytoplasmic vacuolization seems to be the most relevant marker of quality in vitrified oocytes using open carriers. However, the finding in the same oocytes of immature M-SER aggregates as well as the sparse CG with altered ZP and focal microvilli reduction, emphasize the need for further ultrastructural studies, associated with an immunocytochemical approach. It should be also pointed out that the reduction in the number of CG, associated to a zona hardening, implies the use of ICSI as the insemination technique of choice after cryopreservation. This review clearly validates the use of electron microscopy and correlated morphofunctional techniques in the assessment of oocyte cryodamage, also in precise evaluation and refinement of cryopreservation protocols.

\section{References}

1. Smith GD, Motta EE, Serafini P. Theore tical and experimental basis of oocyte vitrification. Reprod Biomed Online 2011;23: 298-306.

2. Gosden R. Cryopreservation: a cold look at technology for fertility preservation. Fertil Steril 2011;96:264-8.

3. Cobo A, Diaz C. Clinical application of oocyte vitrification: a systematic review and meta-analysis of randomized controlled trials. Fertil Steril 2011;96:277-85.

4. Tao T, Del Valle A. Human oocyte and ovarian tissue cryopreservation and its application. J Assist Reprod Genet 2008;25:28796.

5. Herrero L, Martínez M, Garcia-Velasco JA. Current status of human oocyte and embryo cryopreservation. Curr Opin Obstet Gynecol 2011;23:245-50.

6. Lornage J, Salle B. Ovarian and oocyte cryopreservation. Curr Opin Obstet Gynecol 2007;19:390-4.

7. Nazari S, Esmaielzadeh F, Mohsenzadeh M, Khalili MA. Maturation capacity, morphology and morphometric assessment of human immature oocytes after vitrification and in-vitro maturation. Iran J Rep Med 2011;9:209-16.

8. Borini A, Bianchi V. Cryopreservation of mature and immature oocytes. Clin Obstet Gynecol 2010;53:763-74.

9. Kuwayama M, Vajta G, Kato 0, Leibo SP. Highly efficient vitrification method for cryopreservation of human oocytes. Reprod Biomed Online 2005;11:300-8.

10. Antinori M, Licata E, Dani G, Cerusico F, Versaci C, Antinori S. Cryotop vitrification of human oocytes results in high survival rate and healthy deliveries. Reprod Biomed Online 2007;14:72-9.

11. Homburg R, van der Veen F, Silber SJ. Oocyte vitrification-women's emancipation set in stone. Fertil Steril 2009;91: 1319-20.

12. Chian RC, Huang JY, Gilbert L, Son WY, Holzer H, Cui SJ, et al. Obstetric outcomes following vitrification of in vitro and in vivo matured oocytes. Fertil Steril 2009;91: 2391-8.

13 Boldt J. Current results with slow freezing and vitrification of the human oocyte. Reprod Biomed Online 2011;23:314-22. 
14. Liu Y, Du Y, Lin L, Li J, Kragh PM, Kuwayama M, et al. Comparison of efficiency of open pulled straw (OPS) and Cryotop vitrification for cryopreservation of in vitro matured pig oocytes. Cryo Letters 2008;29:315-20.

15. Morató R, Izquierdo D, Paramio MT, Mogas T. Cryotops versus open-pulled straws (OPS) as carriers for the cryopreservation of bovine oocytes: effects on spindle and chromosome configuration and embryo development. Cryobiology 2008;57:137-41.

16. Parmegiani L, Cognigni GE, Bernardi S, Cuomo S, Ciampaglia W, Infante FE, et al. Efficiency of aseptic open vitrification and hermetical cryostorage of human oocytes. Reprod Biomed Online 2011;23:505-12.

17. Khalili MA, Sultan AM, Mojibian M. Role of oocyte morphology on fertilization and embryo formation in assisted reproductive techniques. Middle East Fertil Soc 2005; 10: 72-7.

18. Sathananthan AH, Trounson AO. Effects of culture and cryopreservation on human oocyte and embryo ultrastructure and function. In: Van Blerkom J, Motta PM (eds.) Ultrastructure of human gametogenesis and early embryogenesis. 1989, Kluwer Academic Publ., Boston, pp. 18199.

19. Chen CK, Wang CW, Tsai WJ, Hsieh LL, Wang HS, Soong YK. Evaluation of meiotic spindles in thawed oocytes after vitrification using polarized light microscopy. Fertil Steril 2004;82:666-72.

20. Coticchio G, Sciajno R, Hutt K, Bromfield J, Borini A, Albertini DF. Comparative analysis of the metaphase II spindle of human oocytes through polarized light and high-performance confocal microscopy. Fertil Steril 2010;93:2056-64.

21. Gomes CM, Silva CA, Acevedo N, Baracat E, Serafini P, Smith GD. Influence of vitrification on mouse metaphase II oocyte spindle dynamics and chromatin alignment. Fertil Steril 2008;90:1396-404.

22. Sereni E, Sciajno R, Fava L, Coticchio G, Bonu MA, Borini A. A PolScope evaluation of meiotic spindle dynamics in frozen-thawed oocytes. Reprod Biomed Online 2009;19:191-7.

23. Coticchio G, Bromfield JJ, Sciajno R, Gambardella A, Scaravelli G, Borini A, et al. Vitrification may increase the rate of chromosome misalignment in the metaphase II spindle of human mature oocytes. Reprod Biomed Online 2009;19:29-34.

24. Mandelbaum J, Anastasiou 0, Lévy R, Guérin JF, de Larouzière V, Antoine JM. Effects of cryopreservation on the meiotic spindle of human oocytes. Eur J Obstet Gynecol Reprod Biol 2004;113:S17-23.

25. Ghetler Y, Skutelsky E, Ben Nun I, Ben Dor
L, Amihai D, Shalgi R. Human oocyte cryopreservation and the fate of cortical granules. Fertil Steril 2006;86:210-6.

26. Wu C, Rui R, Dai J, Zhang C, Ju S, Xie B, et al. Effects of cryopreservation on the developmental competence, ultrastructure and cytoskeletal structure of porcine oocytes. Mol Reprod Dev. 2006;73:1454-62.

27. Pellestor F, Anahory T, Hamamah S. The chromosomal analysis of human oocytes. An overview of established procedures. Hum Reprod Update 2005;11:15-32.

28. Gualtieri R, Mollo V, Barbato V, Fiorentino I, Iaccarino M, Talevi R. Ultrastructure and intracellular calcium response during activation in vitrified and slow-frozen human oocytes. Hum Reprod 2011;26:2452-60.

29. Sanchez-Partida LG, Kelly RD, Sumer H, Lo CY, Aharon R, Holland MK, et al. The generation of live offspring from vitrified oocytes. PLoS One 2011;6:e21597.

30. Sathananthan AH, Ng S-C, Bongso A, Trounson A, Ratnam SS. Visual atlas of early human development for assisted reproductive technology. 1993, National University of Singapore and Serono, Singapore.

31. Hyttel P, Vajta G, Callesen H. Vitrification of bovine oocytes with the open pulled straw method: ultrastructural consequences. Mol Reprod Dev 2000;56:80-8.

32. Morató R, Mogas T, Maddox-Hyttel P. Ultrastructure of bovine oocytes exposed to Taxol prior to OPS vitrification. Mol Reprod Dev 2008;75:1318-26.

33. Valojerdi MR, Salehnia M. Developmental potential and ultrastructural injuries of metaphase II (MII) mouse oocytes after slow freezing or vitrification. J Assist Reprod Genet 2005;22:119-27.

34. Nottola SA, Coticchio G, Sciajno R, Gambardella A, Maione M, Macchiarelli G, et al. Ultrastructural changes in human metaphase II oocytes cryopreserved by vitrification. Reprod Biomed Online 2009; 19:17-27.

35. Bonetti A, Cervi M, Tomei F, Marchini M, Ortolani F, Manno M. Ultrastructural evaluation of human metaphase II oocytes after vitrification: closed versus open devices. Fertil Steril 2011;95:928-35.

36. Nottola SA, Macchiarelli G, Coticchio G, Bianchi S, Cecconi S, De Santis L, et al. Ultrastructure of human mature oocytes after slow cooling cryopreservation using different sucrose concentrations. Hum Reprod 2007;22:1123-33.

37. Nottola SA, Coticchio G, De Santis L, Macchiarelli G, Maione M, Bianchi S, et al. Ultrastructure of human mature oocytes after slow cooling cryopreservation with ethylene glycol. Reprod Biomed Online 2008;17:368-77.
38. Gualtieri R, Iaccarino M, Mollo V, Prisco M, Iaccarino S, Talevi R. Slow cooling of human oocytes: ultrastructural injuries and apoptotic status. Fertil Steril 2009;91: 1023-34.

39. Coticchio G, Borini A, Distratis V, Maione M, Scaravelli G, Bianchi V, et al. Qualitative and morphometric analysis of the ultrastructure of human oocytes cryopreserved by two alternative slow cooling protocols. J Assist Reprod Genet 2010; 27:131-40.

40. Sathananthan AH, Trounson A, Freeman L. Morphology and fertilizability of frozen human oocytes. Gamete Res 1987;16:34354.

41. Van Blerkom J, Davis PW. Cytogenetic, cellular, and developmental consequences of cryopreservation of immature and mature mouse and human oocytes. Microsc Res Tech 1994;27:165-93.

42. Coticchio G, De Santis L, Rossi G, Borini A, Albertini D, Scaravelli G, et al. Sucrose concentration influences the rate of human oocytes with normal spindle and chromosome configurations after slow cooling cryopreservation. Hum Reprod 2006;21:1771-6.

43. Borini A, Sciajno R, Bianchi V, Sereni E, Flamigni C, Coticchio G. Clinical outcome of oocyte cryopreservation after slow cooling with a protocol utilizing a high sucrose concentration. Hum Reprod 2006;21:512-7.

44. Yoon TK, Lee DR, Cha SK, Chung HM, Lee WS, Cha KY. Survival rate of human oocytes and pregnancy outcome after vitrification using slush nitrogen in assisted reproductive technologies. Fertil Steril 2007;88:952-6.

45. Combelles CM, Ceyhan ST, Wang H, Racowsky C. Maturation outcomes are improved following Cryoleaf vitrification of immature human oocytes when compared to choline-based slow-freezing. J Assist Reprod Genet 2011;28:1183-92.

46. Ebner T, Moser M, Sommergruber M, Gaiswinkler U, Shebl 0, Jesacher K, et al. Occurrence and developmental consequences of vacuoles throughout preimplantation development. Fertil Steril 2005; 83:1635-40.

47. El Shafie M, Sousa M, Windt M-L, Kruger TF. An atlas of the ultrastructure of human oocytes. 2000, Parthenon Publ., New York.

48. Familiari G, Heyn R, Relucenti M, Nottola SA, Sathananthan AH. Ultrastructural dynamics of human reproduction, from ovulation to fertilization and early embryo development. Int Rev Cytol 2006;249:53141.

49. Mullen SF, Li M, Li Y, Chen ZJ, Critser JK. Human oocyte vitrification: the permeability of metaphase II oocytes to water and 
ethylene glycol and the appliance toward vitrification. Fertil Steril 2008;89:1812-25.

50. Motta PM, Nottola SA, Micara G, Familiari G. Ultrastructure of human unfertilized oocytes and polyspermic embryos in an IVF-ET program. Ann N Y Acad Sci 1988; 541:367-83.

51. Motta PM, Nottola SA, Familiari G, Makabe S, Stallone T, Macchiarelli G. Morphodynamics of the follicular-luteal complex during early ovarian development and reproductive life. Int Rev Cytol 2003;223: 177-288.

52. Makabe S, Van Blerkom J, Nottola SA, Naguro T. 2006. Atlas of human female reproductive function. Ovarian development to early embryogenesis after in-vitro fertilization. Taylor and Francis Publ., London.

53. Dumollard R, Duchen M, Sardet C. Calcium signals and mitochondria at fertilisation. Semin Cell Dev Biol 2006;17: 314-23.

54. Familiari G, Nottola SA, Macchiarelli G, Micara G, Aragona C, Motta PM. Human zona pellucida during in vitro fertilization: an ultrastructural study using saponin, ruthenium red, and osmium-thiocarbohydrazide. Mol Reprod Dev 1992;32:51-61.

55. Jones A, Van Blerkom J, Davis P, Toledo
AA. Cryopreservation of metaphase II human oocytes effects mitochondrial membrane potential: implications for developmental competence. Hum Reprod 2004;19:1861-6.

56. Swain JE, Pool TB. ART failure: oocyte contributions to unsuccessful fertilization. Hum Reprod Update 2008;14:431-46.

57. Nottola S.A., Macchiarelli G. Ultrastructural features and meiotic spindle dynamics in human mature oocytes subjected to different cryopreservation protocols. Proceedings of the 34th National Congress of the Italian Society of Histochemistry, Eur J Histochem 2011;55(Suppl. 1):4. 


\section{Cell cycle checkpoints Methods and protocols} Willis X. Li (ed)

Methods in molecular biology; vol. 782, 2012

Humana Press - Springer Verlag, Heidelberg, Germany

ISBN: 978-1-61779-272-4

Pages: 317; Figures: 68; € 94,95

As it is well known at the end of each cell cycle step there are checkpoints to verify that DNA duplication and segregation (among other events) met every requirements before the cell is allowed to proceed to the next step. Multiple signaling molecules, notably cyclins and the cyclin-dependent kinases (CDKs), play major roles in the cell cycle checkpoint's control. To persuade the reader of the relevance of cell cycle regulation in her/his specific research, I strongly suggest to visit the Nobel prize website (http://www.nobelprize. org/nobel_prizes/medicine/laureates/2001/) and go through the lectures given by Leland $\mathrm{H}$. Hartwell, Tim Hunt and Sir Paul M. Nurse when jointly awarded the Nobel prize in Physiology or Medicine (2001) for their discoveries of key regulators of the cell cycle. By doing this, the reader will get new insights for his researches and will forget the simple idea that the study of cell cycle checkpoints is important just for those need to detect DNA damage, those researchers mainly interested in p16, $\mathrm{Rb}$, Cyclin B / CDK1 and p53 signaling or those studying one of the hallmark of cancer, the cell cycle deregulation. Not quite true, not only those studying tumorigenesis but even all those studying genome integrity (in its several multifacets aspects like genotoxicty, mutagenesis, karyotype rearrangements, stem cell renewal and differentiation to mention a few) will find out how cell cycle sensors, transducers and effectors are molecules embedded in their researches and worthy of investigations. Thanks to nineteen chapters, the book topics span methods and protocols that present several model organisms (yeast, Caenorhabditis, Drosophila, Xenopus, mouse, human) and a mass of techniques (including flow cytometry and indirect immunofluorescence) even for the in vivo live analysis of the cell cycle. Quite interesting to me the chapters devoted to the evaluation of the spindle assembly checkpoint competence in mouse oocytes and that presenting methods to study cancer therapeutic drugs that target cell cycle checkpoints.

CarloAlberto Redi University of Pavia 
Protein expression in mammalian cells

Methods and protocols

James L. Hartley (ed)

Methods in molecular biology; vol. 801, 2012

Humana Press - Springer Verlag, Heidelberg

ISBN: 978-1-61779-351-6

Pages: 287; Figures: 48; €94,95

Twelve pages by Dr. James L. Harley (National Cancer Institute, Frederick, MD, USA; editor of this interesting book) are telling the reader why do we care about protein expression in mammalian cells and the aim of this volume ..... is to provide guidance for those wishing to produce recombinant proteins, and who may be considering doing so in mammals. The reader got immediately the feeling to be guided by a great expert doing protein production in mammalian cells in the every day life (a great merit of the book and a great personal feature of Dr. Hartley): the detailed step by step protocols given in each of the sixteen chapters leaves the reader with the comfortable feeling that he too can afford that goal, when in need. Actually, proteins are fundamental to many aspects of biology and biotechnological considering their place and role in the convergent technologies. The production of proteins thus merges as one of the key features of present day biology. Having said that, I have just to tell the reader that this book is really a great help for all those that wish to enter the field and I am sure even for those who are already involved with recombinant protein production in mammalian cells. I would like to stress that the book is not speaking just for those applying protein production for the biotechnology industry, not at all, the book is intended as well for basic researchers aimed to study native or recombinant proteins to highlight (just to mention a few topics) protein misfolding or the role of proteins in cancer and in general is intended for all those scientists aiming to contribute to solve the many mysteries still connoting the protein world.

How much, with what activities, where and when expressed, how mutated or modified and interacting with what partners are some of the questions that the reader will find well discussed in this well illustrated and well written book; in other words, the reader will find the tools to plane experiments hopefully aiming to get some answers to those questions (always with an applied focus to the production of the proteins). Quite interesting there are few chapters dealing with the Baculoviruses (here Autographa californica), viruses already used in biotechnology for protein expression in insect cells, which are unable to replicate in mammamila cells if not engineered to do this as instructed in these detailed step by step protocols. Transient recombinant protein expressions is discussed in several chapters and in several aspects, quite relevant the reader is told on how to optimize the secretion of antibodies but even on how to improve cytoplasmic expression in mammalian cells.

For those entering the field I have to tell that the gost behind the term mammalian cells has a body which names are embryonic kidney and Chinese hamster ovary cells, the bioreactors able to ensure blockbuster protein productions for commercial biopharma.
CarloAlberto Redi University of Pavia 
Gene expression profiling, $2^{\text {nd }}$ edition

Methods and protocols

Lorraine O'Driscoll (ed),

Methods in molecular biology; vol. $\mathbf{7 8 4}$

2011

Humana Press - Springer Verlag,

Heidelberg, Germany

ISBN: 978-1-61779-288-5

Pages: 210 pp; Figures: 37; €94,95

There must be some good reasons to last for a second edition on the very same topic: likely, the topic is crucial to basic and applied science, it is a very rapid evolving topic and there must occurred some breakthroughs meanwhile the two editions. Well, I think that all of these reasons are here to justify this very wellcome second edition of Gene expression profiling, a topic that is crucial for the understanding of the basic principles of cellular and molecular biology. In other words the study of gene expression profiling actually is the master study from which it depends our ability to understand the gene networks activities keeping alive living organisms and to manipulate their life cycles. The reader will immediately appreciate that the fourteen chapters are written in an extremely detailed manner and the covered themes span the molecular, cellular and histological levels of animal organization in both healthy and patological conditions. It descends that even the techniques highlighted span from advanced PCR protocols (real-time and reverse transcriptase) till the most advanced microscopical methods and techniques (laser scanning confocal and atomic force microscopies) to dissect the relationship existing among networks of networks controlling gene expressions. Interestingly enough, Prof. Lorraine 0'Driscoll (School of Pharmacy, trinity College Dublin) contribute several chapters as coauthor of five among those of high interest: just to mention one of the most challenging situation in which to study gene expression profiles, that of working with minimal amounts of biological reagents, i.e. starting from extracellular material to study gene expression profiling and to be able to get the best results from such specimens. Microarray analysis of mRNAs are obviously a must and actually there are two chapters devoted to explain how the experimental design is crucial to well perform with this methodology and the software and other analytical tools to dataminig the enourmous quantity of data provided by microarray experiments.

The studies of gene expressions in both the most devastating and intricated biological (patological) process (cancer) and those carried out thanks to the use of formalin-fixed and paraffin embedded tissues provide a window over the clinical issues. The microRNAs role in controlling and finely tuning the gene expression profiles is already well known but still the hunt is open to assigne a functional role to many of those already identified in humans (1523 as for 03/11/2012, http://www. mirbase.org): a full chapter is explaining how to perform such studies, in particular considering the intercellular and extracellular microRNAs. The study of the gene expression products cannot be forgot and in fact several chapters are dealing with proteins which are studied by Western blotting, 2D gel electrophoresis and mass spectrometry, tissue microarrays and immunohistochemical techniques.

Manuela Monti
Scientific Department
San Matteo Foundation for Research,
Hospitalization and Health Care,
Pavia, Italy




\author{
Human pluripotent stem cells \\ Methods and protocols \\ Philip H. Schwartz and Robin L. \\ Wesselschmidt (eds) \\ Methods in molecular biology; vol. 767, \\ 2011 \\ Humana Press - Springer Verlag, \\ Heidelberg \\ ISBN: 978-1-61779-200-7 \\ Pages: 487; Figures: 89; €109,95
}

The reader will immediately get the feeling, from the preface of the book, of handling a laboratory manual where no one needed information is missing: the preface itself is a chapter dealing with the historical reconstruction of the paradigmatic breakthroughts occurred in the last few decades in the stem cell world. I appreciated so much this aspect, a preface telling the students of the major achievements and from which prior scientific bases the new discoveries have been fueled, and giving the references of the most relevant papers published ! Prof. Philip H. Schwartz (Center for neuroscience and translational research, Children's hospital of Orange County research institute, Orange, CA) and Robin L. Wesselschmidt (Beckman research institute, City of Hope, Duarte, CA) sketch out in a brief and well written preface their overview of the human pluripotent stem cells world, paying a fitting tribute to the previous mouse achievements and telling the reader how they conceived the book structure. Which architecture appear very well designed. The volume is a compilation of 33 detailed protocols divided in six parts, logically following one another from the laboratory essentials (design, equipment, stem cells banking, etc.) in part one, through part two devoted to illustrate protocols for the derivation of stem cells (five chapters, dealing with the derivation from both embryos and the use of retrotransfection of stemness genes to induce terminally differentiated cells to become pluripotent; not forgetting the use of piggyBac transposones to avoid the risks of retrotransfection) and part three presenting the growth, maintenance and expansion of stem cells. Quite interesting the attention payed to illustrate how to culture human pluripotent stem cells in xeno-free media in order to match the cell factory's requirement for good manufactural practices to produce the cells needed for therapeutic applications. Once produced the desired cells must be characterized (part four), a procedure that can be accomplished thanks to several protocols (hopefully by the use of many of them) spanning from the classical karyotyping, FISH, flow and immunocytochemical techniques to the use of microarray technology to profile gene expressions and the epigenome to the most advanced quantitative proteomic analysis of human pluripotent stem cells. Eventhough the present day use of human pluripotent stem cells is still related to the 1998 J.A. Thomson statement that these cell lines should be useful in studying human developmental biology, drug discovery and transplantation medicine, a great hope is that to be able to use them for rigenerative medicine through cellular therapies, to meet intractable diseases. To reach this goal a necessary prerequiste is the genetic manipulation (part five) of these cells to target specific gene expression or, viceversa, to get episomal transgene expressions. The generation of specific cell types (part six) useful for the possible treatment of several diseases (apart the hematological ones which have a long tradition of cures based on multipotent stem cells) is highlighted considering those diseases that seems much more at the hand: Parkinson disease and the treatment of the necrotic tissue due to infarction.

All the protocols are superbly illustrated with many figures and charts presented in full colours. I think this book is a must for all those are working with human pluripotent stem cells, no matter if students entering the field or colleagues already involved, each of them will get the chance to sharpen their conceptual insights.

CarloAlberto Redi University of Pavia 